\title{
INVESTIGATIONS OF DIRECTOR FIELD ARRANGEMENT INDUCED IN TUNABLE LIQUID CRYSTALLINE WAVEGUIDE
}

\author{
A. WALczak \\ Institute of Applied Physics, Military University of Technology \\ Kaliskiego 2, 01-489 Warsaw, Poland \\ (Received July 15, 1998; in final form October 20, 1998)
}

\begin{abstract}
Measurements of the refractive index profile have been made to provide data for obtaining local optical axis arrangement in the waveguide cross-section. Liquid crystalline planar waveguide tuned with the electric field has been used. A new approach is proposed to accomplish refractive index profile measurements in a direct way. The modal line method is presented as a tool that allows one to do it and to improve the attenuated total reflection disadvantages as well. The obtained results maintain applicability of the modal line method for a direct refractive index profile measurement. To verify the method, nematic 6 CHBT (4-trans-4- $n$-hexyl-cyclohexyl-isothiocyanatobenzene) described earlier in the literature has been examined. A nematic mixture W602 contained 6CHBT as a main component has been examined too.
\end{abstract}

PACS numbers: $61.30 . \mathrm{Gd}$

\section{Introduction}

In the literature a few quite different methods for refractive index profile (RIP) measurements in liquid crystals have been presented [1]. The RIP investigated in liquid crystal (LC) has been calculated in an indirect way from reflectivity on liquid crystalline layer, measured in attenuated total reflection (ATR) experiments [2]. The reflectivity is measured with the aid of the prism coupler. After that, the fitting of the measured and theoretically obtained reflectivities supplies RIP values [3, 4]. During theoretical calculation one assumes that LC layer is considered as a multi-layered system of uniform biaxial slabs of dielectric material, with each slab thickness of $d \ll \lambda$, where $\lambda$ is the wavelength of the incident light [5]. An algorithm of the calculation is usually built on the transfer matrix routine developed by Berreman and Scheffer and Azzam and Bashara [6]. In the presence of the $s p$ mode mixing, this algorithm often exhibits numerical instabilities in reflectivity calculation.

One knows that thin liquid crystalline layer properties are strongly dependent on liquid crystal - substrate interactions. Especially LC layer alignment is determined by that interaction. The area of the LC layer that is affected by the considered interaction is of $0.1-1 \mu \mathrm{m}$ thickness when it is measured from the layer edge. The director field orientation in this area can be different from that in the layer bulk. Because of different tilt angle in boundary layer from that in the bulk, 
dividing into equal sublayers in ATR calculations is a source of mistakes. On the other hand, there is no way to know a priori a thickness of that boundary layer. Those disadvantages can be overcome by modal line method (m-line method) that allows one to make a direct RIP measurement in the LC waveguides [7]. That method has been developed earlier in fiber optics $[8,9]$ and is adapted here to liquid crystal investigations.

The local director field orientation inside liquid crystal film has been investigated also by Simoni et al. [10] by means of total internal reflection (TIR) from liquid crystalline film deposited on the bottom of the prism. The authors expected to obtain several fringes coming from the interference between wave reflected at the entrance surface and the wave reflected by TIR inside the sample. In fact that method is very similar to the leaky guided mode method exploited by Sambles and co-authors $[2,5,7]$. When the LC film is heterogeneous then the rules used by Simoni et al. [10] are fulfilled only for an ordinary wave. Further development of that method is presented in Warenghem et al. [11] and references therein. Nevertheless only the local tilt can be estimated in that method.

The aim of this paper is to develop a new approach for determining a local optic axis position in the LC layer cross-section. If RIP in the LC layer is known in two perpendicular cross-sections then a tilt of a local optical axis can be determined [12]. In such a way one can obtain director field orientation in the LC layer. The results have been shown in dependence on external electric field rms voltage. The tilt angle in all figures is shown as measured between director and waveguide planes.

\section{Experimental set-up and modal line method}

Let us consider a planar waveguide (shown in Fig. 1) in which the refractive index is a function $n(x)$ where $x$ is perpendicular to the guiding layer. The total phase change along zig-zag of the beam path must be a multiple of $2 \pi$ for wave propagation in the guide. This resonance condition is [13]

$$
2 k_{0} \int_{0}^{x_{m}}\left[n^{2}(x)-N_{m}^{2}\right]^{1 / 2} \mathrm{~d} x+2 \phi_{c a}+2 \phi_{c b}=2 \pi m .
$$

The turning point $x_{m}$ is defined by $n\left(x_{m}\right)=N_{m}$, where $N_{m}$ is the effective refractive index for a mode that propagates along the guide. In (1) $m$ is an integer numbering of the modes, and $k_{0}$ is the wave vector in free space. Phase changes $\phi_{c a}$ and $\phi_{c b}$ are described by Fresnel rules [13]

$$
\phi_{c a, c b}=\arctan \left[\frac{n\left(x_{m}\right)}{n_{a, b}}\right]^{\rho} \sqrt{\frac{N_{m}^{2}-n_{a, b}^{2}}{n^{2}\left(x_{m}\right)-N_{m}^{2}}} .
$$

Here $\rho$ equals 0 for a wave polarized perpendicularly to the plane of incidence or 1 for a wave polarized in plane of incidence. The symbols $a, b, c$ assign the waveguide regions, where $c$ is the region of a guide, while $b$ and $a$ are the regions of optical buffer on both boundaries of the waveguide.

To resolve Eq. (1) the effective indice $N_{m}$ for a mode with the number $m$ has to be measured. Such reconstruction of $n(x)$ is an inverse problem and a solution 


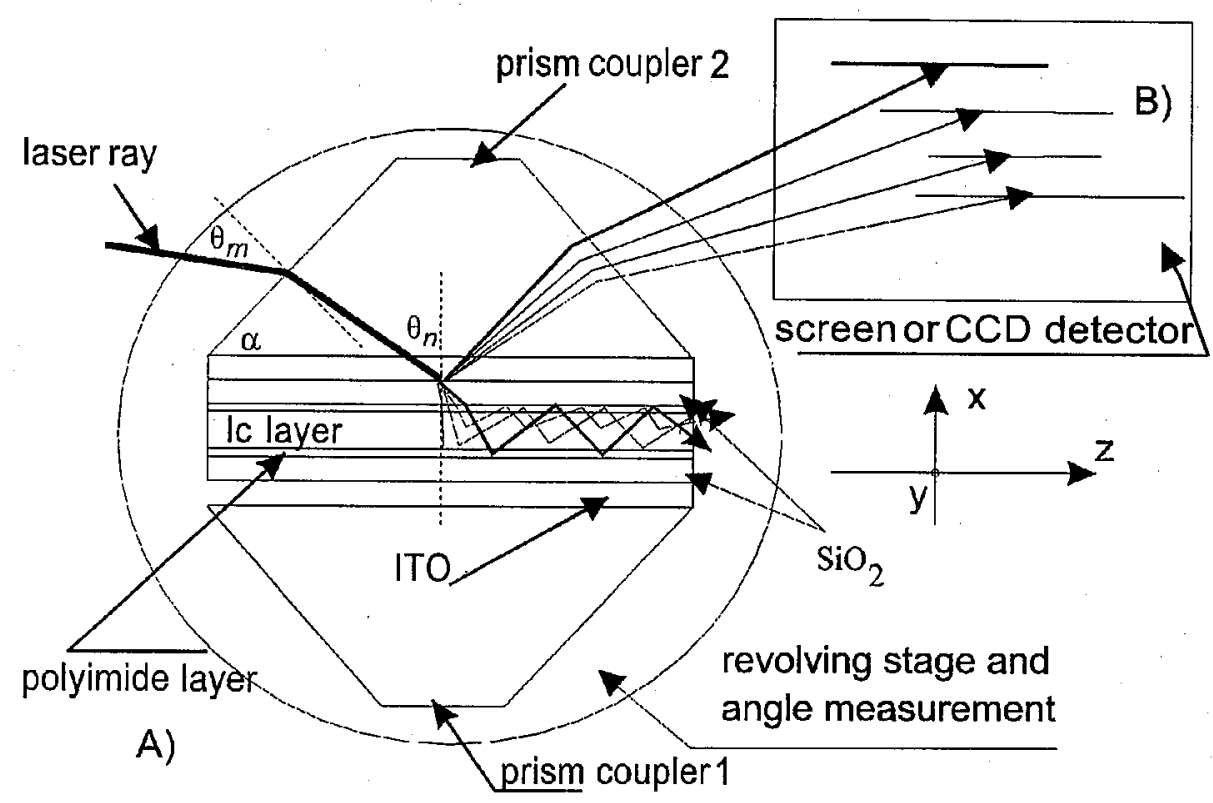

Fig. 1. The measurement device cross-section (A) and scheme of the modal line visualization (B) $(x, y, z$ are Cartesian coordinates).

has been obtained by WKB method [12]. In the first term of the product in (1) we assumed a linear variation [12]

$$
n(x)=N_{k}+\left(\frac{N_{k-1}-N_{k}}{x_{k}-x_{k-1}}\right)\left(x_{k}-x\right) .
$$

Integrating in (1) with the above assumptions results in recursive relation for $x_{m}$ and $x_{m-1}$ in which in the first step we can assume that $x_{0}=0, N_{0}=n(0)$

$$
\begin{aligned}
& \ln \left(x_{m}-x_{m-1}\right)=-\frac{1}{2} \ln \left[N_{m}\left(N_{m-1}-\sqrt{N_{m-1}^{2}-N_{m}^{2}}\right)\right] \\
& \quad+\frac{N_{m-1}-N_{m}}{N_{m}^{2}}\left(m+\frac{1}{2}\right) \pi-\frac{N_{m-1}-N_{m}}{N_{m}^{2}} \sum_{k=1}^{k=m-1} \frac{1}{2\left(N_{k-1}-N_{k}\right)} \\
& \quad \times\left\{\left(x_{k-1}-x_{k}\right)\left(N_{k} \sqrt{N_{k}^{2}-N_{m}^{2}}\right)\right. \\
& +N_{m}^{2} \ln \left[\left(N_{k}-\sqrt{N_{k}^{2}-N_{m}^{2}}\right)\left(x_{k}-x_{k-1}\right)\right] \\
& \quad+\left(x_{k}-x_{k-1}\right)\left(N_{k-1} \sqrt{N_{k-1}^{2}-N_{m}^{2}}\right) \\
& \left.\quad+N_{m}^{2} \ln \left[\left(N_{k-1}-\sqrt{N_{k-1}^{2}-N_{m}^{2}}\right)\left(x_{k}-x_{k-1}\right)\right]\right\} .
\end{aligned}
$$


Each $N_{m}$ is the discrete value of $n\left(x_{m}\right)$ so one can fit as many interpolating polynomial parameters as mode number. The trial function $n(x)$ obtained in such a way is used in (1) to calculate corrections for turning points. Those corrections are used in (4) to verify turning point positions. It is repeated until one obtains a demanded accuracy for turning points.

The effective indices have been measured in a device, the scheme of which has been shown in Fig. 1. It is constructed as follows: the liquid crystal layer is deposited between two prisms of the high refractive index. The bottom of each prism is coated with layers, refractive index of which is lower than the one of the liquid crystal. The indium tin oxide (ITO) is a part of the buffer layer to apply the voltage through the layer. The $\mathrm{LC}$ is aligned with a rubbed polyimide covering the $\mathrm{SiO}_{2}$, layer, which is deposited on the surface of ITO. The area of ITO, $\mathrm{SiO}_{2}$ and polyimide create an optical buffer. The thickness of this optical buffer layer is properly chosen to ensure the light coupling into the waveguide. A symmetrical construction of the measuring device allows one to examine LC layers with non-symmetric RIP.

If an incident photon along the interface between prism and LC layer matches the momentum of the optic mode in the waveguide, the refractive index for the mode excited at this moment is equal to

$$
N_{m}=n_{p} \sin \left[\alpha+\arcsin \left(\frac{\sin \left(\Theta_{m}\right)}{n_{p}}\right)\right] .
$$

This relation can be easily seen from Fig. 1. The used symbols assign $\alpha-$ the prism's angle as in Fig. 1, $\Theta_{m}$ - the incidence angles during the guided mode excitation, $n_{p}$ - the refractive index of the prism. So $N_{m}$ from formula (1) can be easily measured by determination of the $\theta_{m}$ angle and can be applied in (1).

Both prisms in the measurement device have been worked as input-output couplers. Consequently evanescent field from the excited modes is coupled into the prism and can be observed as modal lines on the screen or CCD camera (see Fig. 1B).

When the RIP is obtained from the solution of (1) the known rule for birefringent media

$$
n_{\mathrm{eff}}(\gamma)=\frac{n_{\mathrm{e}} n_{\mathrm{o}}}{\sqrt{n_{\mathrm{e}}^{2} \sin ^{2} \gamma+n_{\mathrm{o}}^{2} \cos ^{2} \gamma}}
$$

provides a possibility to determine an angle $\gamma$ between the local optical axis and the wave vector $k$ of the guided mode. In formula (6) $n_{\mathrm{e}}$ and $n_{\mathrm{o}}$ are the extraordinary and ordinary refractive indices for the applied liquid crystal. The meaning of angle $\gamma$ is illustrated in Fig. 2.

In twisted nematic (TN) the extraordinary wave vector does not remain in plane of incidence during wave propagation. The propagation constant of the extraordinary wave is dependent on angle between optical axis and wave vector [14]. So in a twisted structure formula (6) cannot be used for the obtained angle $\gamma$. An ordinary wave has the propagation constant independent of that angle.

An accurate determination of the angle between the wave vector of the measured mode and the optical axis in the turning point allows one to operate in the 


$$
\frac{\theta}{\theta}
$$




\section{Results of measurements}

The prisms in a measurement device have been made from $\mathrm{Bi}_{12} \mathrm{SiO}_{20}$ as square based (with faces $12 \mathrm{~mm} \times 12 \mathrm{~mm}$ ). Both prisms worked as input and output couplers. The laser He-Ne was applied in the measurements. The prism bottoms have been coated with the tin-indium oxide and with $100 \mathrm{~nm}$ thick $\mathrm{SiO}_{2}$. On this optical buffer the covering rubbed polyimide layer has been deposited $(\approx 40 \mathrm{~nm})$. At $632.8 \mathrm{~nm}$ wavelength the refractive index of the prism is equal to 2.5450 while the $\mathrm{SiO}_{2} / \mathrm{ITO} /$ polyimide optical buffer's refractive index has been measured as equal to 1.4850 .

The homogeneous layers of two nematic liquid crystal (NLM), mixture W602 (catalogue number from AWAT in MUT Poland), and 6CHBT from that laboratory have been examined in the experiment. Values of the refractive indices in this material are equal to $n_{\mathrm{e}}=1.6494$, and $n_{\mathrm{o}}=1.5029$ in W602, and $n_{\mathrm{e}}=1.6800$, and $n_{\mathrm{o}}=1.5200$ for $6 \mathrm{CHBT}$ when $T=20.0^{\circ} \mathrm{C}$ [15-17].

Let us analyse homogeneous alignment of NLC for two different cases. The first case, when directors are aligned in plane parallel to the waveguide axis (along the $z$ axis in Fig. 1), and the second, when directors lie in plane perpendicular to the waveguide axis (parallel to the $y$ axis in Fig. 1). In the second case mixed $s-p$ modes are always present in the waveguide [12]. In that case the wave vectors of both ordinary and extraordinary waves are perpendicular to the optic axis. Hence $n_{\text {eff }}$ does not change while voltage increases (see Fig. 5), only a small change in LC-polyimide boundary is observed. It can be interpreted as a result quite different from that in the layer bulk LC-substrate interaction in the layer boundary vicinity. The refractive index profile for this case is illustrated in Fig. 5.

In the first case TE modes do not couple with TM modes. The wave vector for ordinary and extraordinary waves lies in the plane of incidence and simultaneously

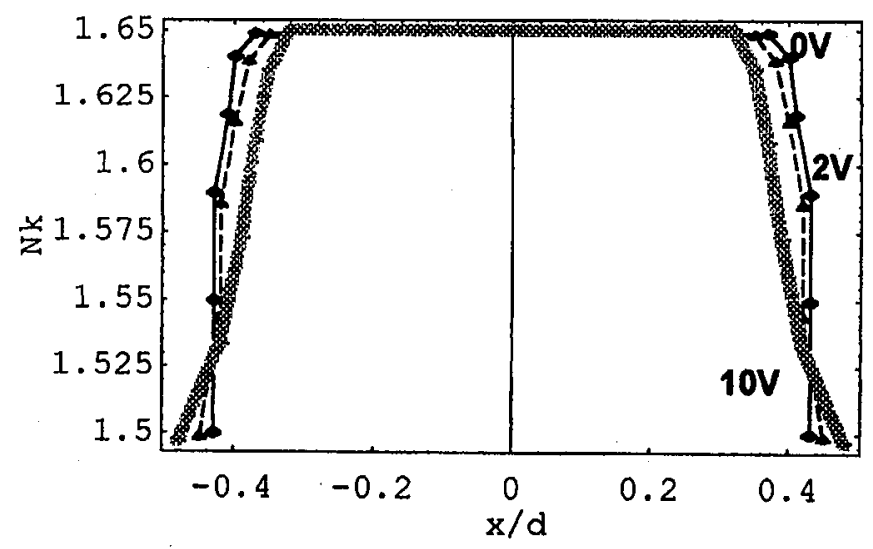

Fig. 5. Values of RIP in turning points for voltage $U=0 \mathrm{~V}, U=2 \mathrm{~V}, U=10 \mathrm{~V}$ in initially homogeneous W602 layer; initial director alignment perpendicular to the waveguide axis; values of $n_{\text {eff }}$ measured at turning points for $s p$ modes. Experimental points joined with line just to distinguish different profiles (layer thickness $d=6 \mu \mathrm{m}$ ). 


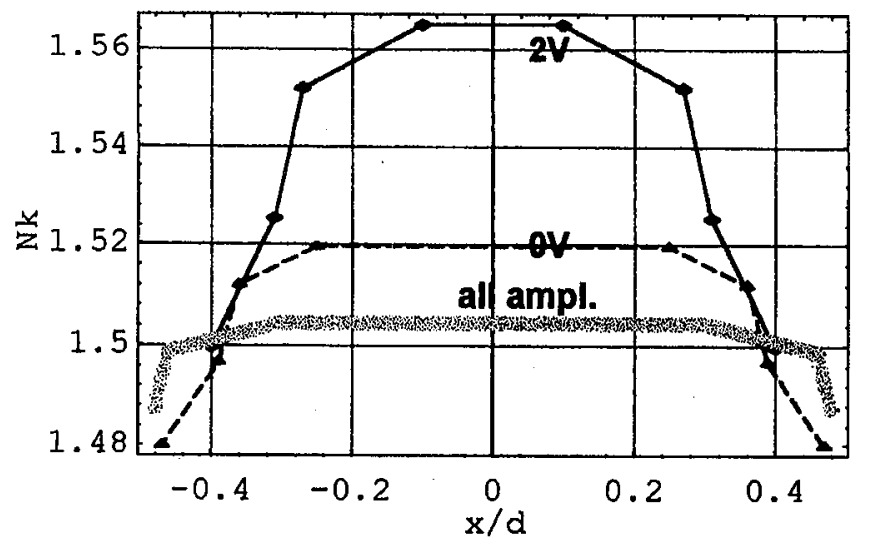

Fig. 6. Values of RIP in turning point at voltages $U=0 \mathrm{~V}$ and $U=2 \mathrm{~V}$; initially homogeneous W602 layer; initial director alignment along waveguide, $n_{\text {eff }}$ values measured at the turning points for $s$ modes (upper curves) and $p$ modes (lowest curve) in the LC waveguide. Experimental points joined with line just to distinguish different profiles (layer thickness $d=6 \mu \mathrm{m}$ ).

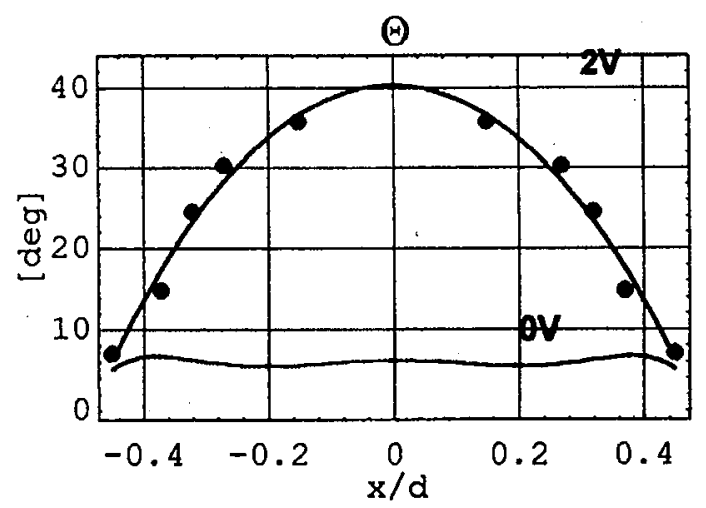

Fig. 7. The induced tilt angle values in electrically deformed homogeneous W602 layer with a thickness $d=6 \mu \mathrm{m}$ (initial alignment along $z$-axis). Experimental values at the turning point for voltage equal to $2 \mathrm{~V}$ are shown.

in the plane parallel to the guide axis. The $p(\mathrm{TE})$ mode is equivalent to ordinary wave and is unchanged during electric dcformation of the waveguide layer. The $s$ (TM) mode is an extraordinary wave and those modes are highly sensitive to external field voltage. The result of the measurement for that case is presented in Fig. 6.

We have assumed that the conduction processes may be neglected because of:

1. the resistivity is equal to $10^{12} \Omega \mathrm{cm}$ in liquid crystal,

2. the highest used voltage is equal to $10 \mathrm{~V}$ in the cell $6 \mu \mathrm{m}$ thick,

3. the external field frequency is equal to $2 \mathrm{kHz}$. 
It means that the ion transport does not influence the orientation of the LC molecules in on state. The variations in RIP for a voltage $U=10 \mathrm{~V}$ shown in Fig. 4 may be associated with the deformation induced in the boundary area which can be initially inhomogeneous because of substrate roughness.

The tilt angle across the layer for initially homogeneous W602 layer has been shown in Fig. 7. The tilt values have been calculated from the angles obtained in formula (6).

Approximation deviations can be seen in Fig. 7 so just joining line for experimental data like in Fig. 5 and Fig. 6 seems to be quite appropriate for certäin presentations. Profiles of the refractive index in thin $3 \mu \mathrm{m}$ thick layer of $6 \mathrm{CHBT}$ are shown in Fig. 8. The behaviour of director tilt for illustrated cases is shown in Fig. 9.

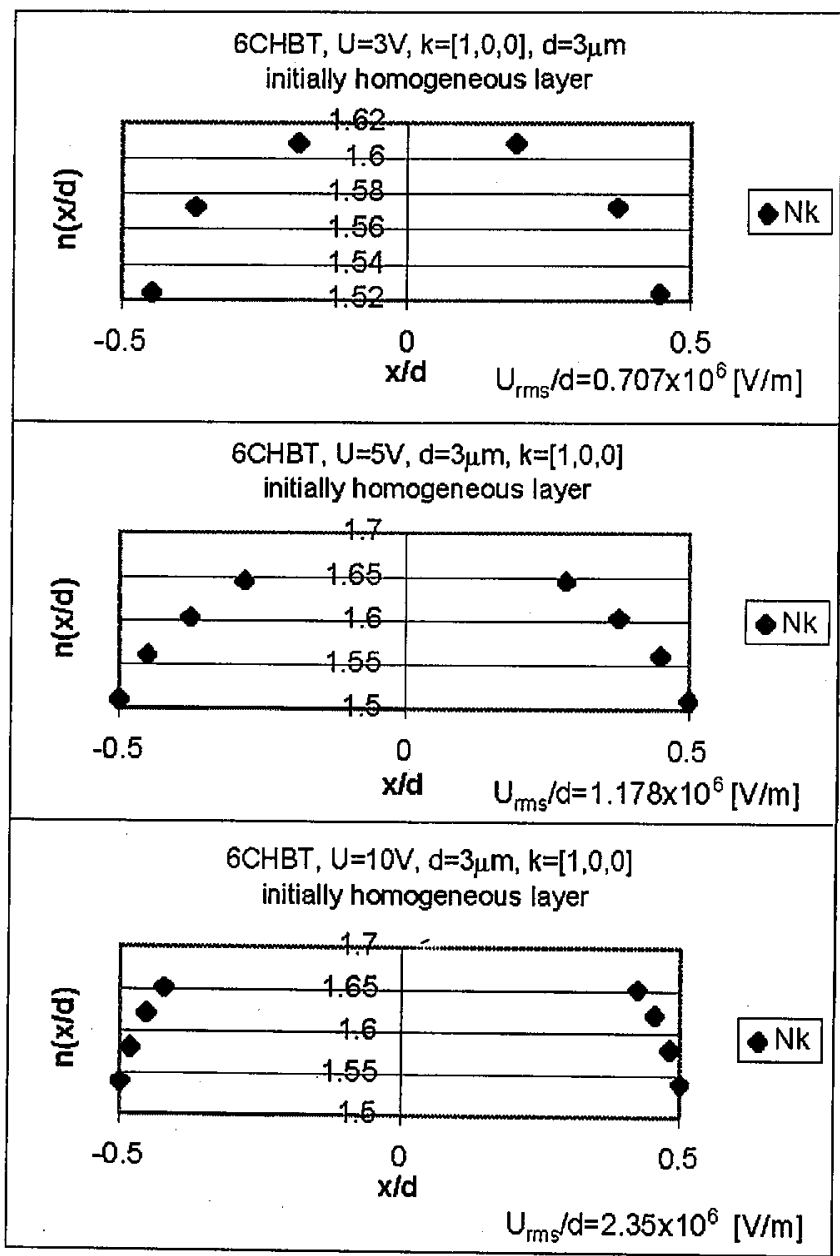

Fig. 8. The refractive index profile in a $3 \mu \mathrm{m}$ thick $6 \mathrm{CHBT}$ layer shown in turning points across the layer for different voltages of the external field. 


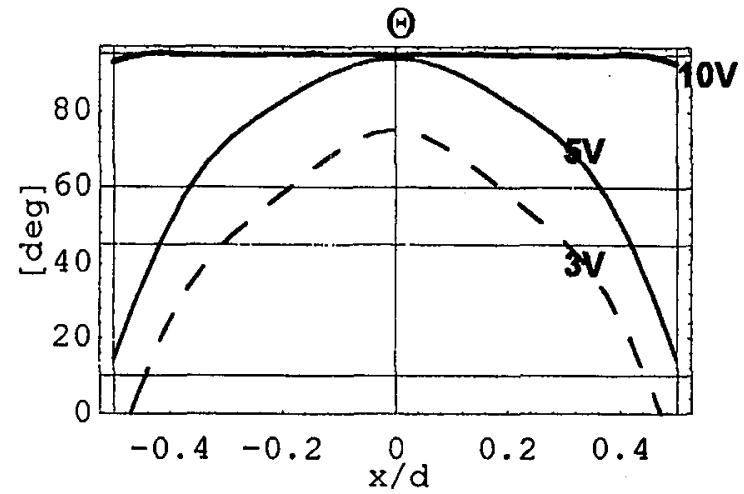

Fig. 9. Approximated director tilt across the layer for different voltages of the external field in a $3 \mu \mathrm{m}$ thick $6 \mathrm{CHBT}$ layer.

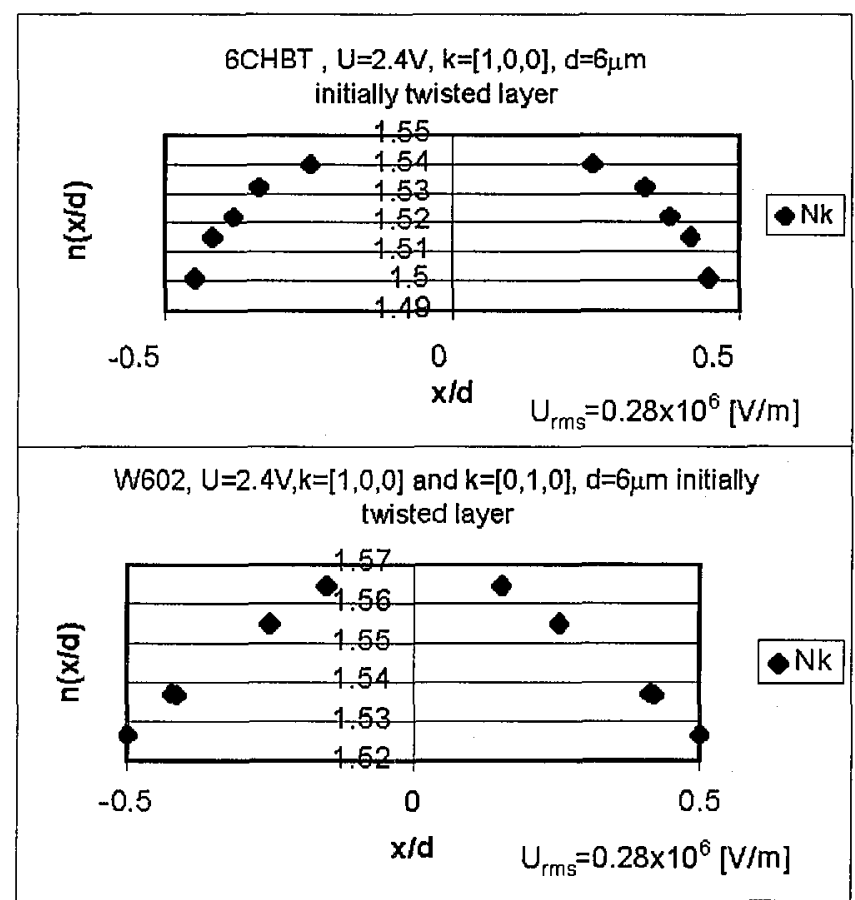

Fig. 10. Refractive index profile values in turning points for 6CHBT and W602.

For the same voltage, layer thickness and initial layer alignment we get comparison of the investigated liquid crystalline substances. Below (Fig. 10) RIP in initially twisted $3 \mu \mathrm{m}$ thick layer for both of them is shown. In Fig. 9 we can see that a certain area in the layer boundary vicinity has not been deformed when 
$U=3 \mathrm{~V}$. It may be caused by numerical inaccuracies during polynomial approximation. A similar situation has been illustrated in Fig. 7. Because the profiles in Fig. 10 exhibit a lack of deformation in part of the layer as a consequence of measurement we can suppose that area which is not deformed in the 6CHBT layer is a real result. The explanation is that $6 \mathrm{CHBT}$ molecules are strongly anchored. Simultaneously W602 exhibits a lower deformation than 6CHBT. It means that the anchoring energy of the nematic mixture is lower than that in 6CHBT, but the elastic constants should be higher in W602.

\section{Conclusions}

The advantage of the presented method is that RIP is directly measured and no aid of theoretical models of LC layer deformation is needed. The used formulae give values of refractive index in turning points for each mode guided in the LC layer. Those points lie on the refractive index profile. Data of a RIP obtained in such a way can be applied for local optical axis investigations in the LC layer cross-sections. The presented results have been obtained with an accuracy of about $2 \%$ for turning point position and $0.5 \times 10^{-3}$ for refractive index value. The achievable accuracy can be improved. One should remember that an integer in formula (1) is taken over effective thickness of the waveguide. It results in different errors obtained for turning point position of each mode. The biggest error is for the first mode with an internal angle of reflection near the critical angle. The values obtained for the first mode (with the lowest index $m$ ) should be rejected during RIP calculation. Another disadvantage is that the m-line method can be applied only for multimode waveguides.

The presented method allows one to observe deformations in liquid crystalline layers and to verify theoretical model of such deformations. Because in the aligned liquid crystals the local optical axis is parallel to the director, one can assume that the obtained results are director field shape in the considered LC layers.

\section{Acknowledgments}

This work is supported through grant of the Committee for Scientific Research No. 7T08C00712. The author is grateful to Dr. E. Nowinowski-Kruszelnicki for his inspired comments and participation in the LC layers preparation and to Dr. A. Kiezun for very helpful discussion of the optical measurements.

\section{References}

[1] G.L. Yip, P.C. Noutsios, L. Chen, Appl. Optics 35, 236 (1996).

[2] Fuzi Yang, J.R. Sambles, J. Opt. Soc. Am. B 10, 858 (1993).

[3] D.Y.K. Ko, J.R. Sambles, J. Opt. Soc. Am. 5, 1863 (1988).

[4] D.J. Mikulin, D.A. Coley, J.R. Sambles, Liq. Cryst. 22, 301 (1997).

[5] K.R. Welford, J.R. Sambles, Liq. Cryst. 2, 91 (1987).

[6] D.W. Berreman, T.J. Scheffer, Phys. Rev. Lett. 25, 2135 (1968); R.M.A. Azzam, N.M. Bashara, Ellipsometry and Polarised Light, North-Holland, Amsterdam 1979, p. 34. 
[7] C.R. Lavers, J.R. Sambles, Liq. Cryst. 8, 577 (1990).

[8] A. Walczak, E. Nowinowski-Kruszelnicki, A. Kieżun, L.R. Jaroszewicz, Elec. \&Telecom. Quarterly PAS 4, 42 (1996).

[9] D. Marcuse, IEEE J. QE QE-9, 1000 (1973).

[10] F. Simoni, F. Bloisi, L. Vicari, M. Warenghem, M. Ismaili, D. Hector, Europhys. Lett. 21, 189 (1993).

[11] M. Warenghem, D. Louvergneaux, F. Simoni, Mol. Cryst. Liq. Cryst. 282, 235 (1996).

[12] A. Walczak, E. Nowinowski-Kruszelnicki, A. Kieżun, SPIE Proc. 3318, 344 (1997).

[13] M.K. Barnoski, Introduction to Integrated Optics, Plenum Press, New York 1974, p. 27.

[14] F. Ratajczyk, Optics of Anisotropic Media, PWN, Warszawa 1994 (in Polish).

[15] R. Dąbrowski, J. Dziaduszek, T. Szczuciński, Mol. Cryst. Liq. Cryst. 192, 31 (1990).

[16] Z. Raszewski, J. Kędzierski, J. Rutkowska, J. Żmija, R. Dąbrowski, T. Opara, Proc. SPIE Int. Soc. Opt. Eng. 1845, 325 (1992).

[17] R. Buchecker, M. Schadt, Mol. Cryst. Liq. Cryst. 149, 359 (1984). 